\title{
Análisis de la demanda en Salud Mental en el Principado de Asturias: Características de la demanda y respuesta asistencial.
}

Mental Health demand analysis in the Principality of Asturias (I): Demand characteristics and assistance provided.

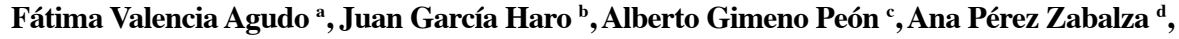 \\ María Eugenia de la Viuda Suárez ${ }^{\mathrm{e}}$.
}

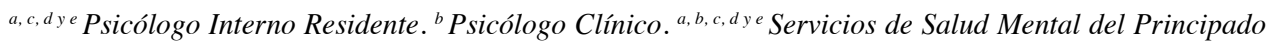
de Asturias. Asturias, España.

Correspondencia: Fátima Valencia Agudo (fatima-valencia@hotmail.es)

Recibido: 05/09/2013; aceptado con modificaciones: 15/04/2014

\begin{abstract}
RESUMEN:Introducción. En los últimos años ha aumentado el número de demandas a Salud Mental relacionadas con Trastornos Mentales Comunes y códigos Z. El presente trabajo analiza las características de la demanda y el tipo de respuesta asistencial en tres Centros de Salud Mental del Principado de Asturias.

Método. Se trata de un estudio descriptivo. De las derivaciones realizadas a estos tres Centros de Salud Mental se recogen una serie de variables sociodemográficas, clínicas, relacionadas con la demanda y relacionadas con la respuesta asistencial.

Resultados y conclusiones. Se detecta un $68,8 \%$ de casos que podrían corresponder con Trastornos Mentales Comunes. Se asigna un $59,1 \%$ de los pacientes a psiquiatra y un $39,7 \%$ a psicólogo clínico. Los casos de depresión, trastorno adaptativo y trastorno ansioso-depresivo son asignados en mayor medida a psiquiatra, mientras que no hay diferencias en el caso de la ansiedad. Estos datos no coinciden con las recomendaciones de las guías clínicas. Tras observar los resultados se plantea la importancia de la figura del psicólogo clínico, tanto en atención primaria como en especializada.

PALABRAS CLAVE: Análisis de la Demanda, Atención Primaria, Salud Mental, Psicología Clínica, Trastornos Mentales Comunes.
\end{abstract}

ABSTRACT: Background. In recent years, the number of referrals to Mental Health Services related to Common Mental Health Disorders and $\mathrm{Z}$ codes has increased. This project analyses the characteristics of these referrals and the assistance provided in three Mental Health Centers in Asturias.

Method. We collected sociodemographic and clinical variables from the referrals to the Mental Health Centers. In addition, we compiled data related to the referral process and to the assistance provided.

Results and conclusions. It was found that $68,8 \%$ of the sample might fall into the category of Common Mental Health Disorders. Moreover, 59,1\% of the patients were assigned to a psychiatrist and $39,7 \%$ to a clinical psychologist. Depression, adjustment disorders and anxiety-depressive disorders were assigned more frequently to a psychiatrist, whereas there were no differences regarding anxiety. The data do not match the clinical guidelines. Taking these results into account, we discuss the important role of the clinical psychologist, both in Primary Health Care and in Mental Health Services.

KEY WORDS: Demand Analysis, Primary Health Care, Mental Health, Clinical Psychology, Common Mental Health Disorders. 
ORIGINALES Y REVISIONES

\section{Introducción}

En el año 1983 comienza el desarrollo de la reforma psiquiátrica en Asturias, que supondrá la reconversión del Hospital Psiquiátrico en la Red de Servicios de Salud Mental Comunitarios, integrada por Centros de Salud Mental (CSM en adelante), Unidades de Hospitalización Psiquiátrica Breve (UHP), Unidades de Tratamiento de Toxicomanías (UTT), Hospitales de Día (HD) y Comunidades Terapéuticas (CT).

La adjetivación de comunitario aplicada a la Salud Mental implica que la intervención en este ámbito se realiza en la comunidad en la que el individuo vive, sin desinsertarlo de la misma. En este sentido, los CSM supondrían el pilar fundamental de la atención ambulatoria y por tanto la base del modelo comunitario. Por dicho motivo, este artículo se centra en la labor asistencial de dicho dispositivo.

En los últimos años se ha producido un cambio en el patrón de utilización de los servicios públicos de Salud Mental, con un incremento notable de nuevas demandas por parte de la población. Una parte importante de estas cumplen criterios relacionados con los trastornos neuróticos y reacciones de adaptación, englobados en la denominación de Trastornos Mentales Comunes (1). En Asturias, según datos de primeras consultas, este tipo de demandas pasaron del 25,5\% en 1989 (2) al $56,58 \%$ en 2000 (3) y al 60\% en 2005 (4). Otros casos en cambio no cumplen criterios diagnósticos para trastorno mental. Son demandas que se relacionan con sentimientos de malestar no patológicos derivados de los avatares de la vida cotidiana y que en las clasificaciones nosográficas actuales no constituyen ningún diagnóstico psicopatológico. En un estudio realizado en Madrid, un 24,4\% de los sujetos que acuden a un Centro de Salud Mental (CSM) no presentan ningún trastorno mental diagnosticable según criterios de la CIE-10 (5). Los sistemas clasificatorios actuales recogen este tipo de demandas en una categoría separada, fuera de los trastornos mentales. En la CIE-10 se denominan códigos Z y en el DSM-IV-TR se recogen en el eje IV con el nombre de problemas psicosociales y ambientales. Además, en el estudio citado la mitad de estas demandas acuden a los Servicios de Salud Mental con tratamiento farmacológico ya instaurado y de estos, el 53\% lleva menos de dos meses con él pautado. Esto implica que tratamiento y derivación se realizan al mismo tiempo o con un lapso temporal muy reducido; no se espera siquiera a conocer el efecto del tratamiento antes de derivar (5). Finalmente, los autores plantean que alrededor de uno de cada cinco códigos $\mathrm{Z}$ ha sido derivado de forma preferente cuando este tipo de derivaciones teóricamente está reservada para pacientes con especial gravedad clínica que han de ser atendidos lo antes posible.

Esta realidad en la red de Salud Mental tiene importantes repercusiones:

- Elevada carga asistencial.

- Aumento del intervalo entre consultas. 
ORIGINALES Y REVISIONES

- Incremento de la duración de la atención para todos los trastornos, tanto para los de previsible evolución prolongada como para los de corta evolución.

- Detrimento en la atención a los trastornos mentales más graves.

- Aumento de los costes económicos directos (consultas, pruebas diagnósticas, gasto farmacéutico, etc.) y los indirectos (bajas laborales, invalidez, bajo rendimiento, etc.).

Todo esto lleva a dos cuestiones fundamentales: la definición y aplicación de criterios de derivación y tratamiento por parte de los derivantes, en concreto de los Médicos de Atención Primaria (MAP), y otra, la necesidad de dar una respuesta adecuada a las demandas, en términos de efectividad y eficiencia.

Cabe plantearse cómo conseguir proporcionar una buena atención a los trastornos mentales comunes y códigos $\mathrm{Z}$, que constituyen una demanda social y son susceptibles de ser psicopatologizados y medicalizados (y por tanto cronificados), a la par que prestar la atención que necesitan los trastornos mentales más graves. Una posible solución sería modificar el tipo de atención que se está prestando en la actualidad, basada en el tratamiento psicofarmacológico. Conviene recordar que entre un $18 \%$ y un $39 \%$ de los problemas atendidos en Atención Primaria están relacionados con la Salud Mental (6) y que la respuesta asistencial en este nivel está siendo mayoritariamente farmacológica. De acuerdo con gran cantidad de investigaciones y revisiones, las intervenciones psicológicas suponen un tratamiento eficaz, efectivo y más eficiente que los psicofármacos a la hora de intervenir en una amplia gama de trastornos psicológicos (7-9), incluida la depresión mayor (10). Tiene la ventaja añadida de tener limitados efectos secundarios y de mantener sus efectos a largo plazo, reduciéndose por tanto recaídas tras la finalización del tratamiento. La psicoterapia es según guías clínicas una intervención de elección en el caso de trastornos de ansiedad (11-13). Por otra parte, se plantea que la psicoterapia ha de ofrecerse a pacientes con depresiones leves y moderadas, depresiones reactivas y trastornos adaptativos; asimismo se recomienda su introducción en depresiones graves y de larga evolución ya que su eficacia a corto plazo es similar a la del tratamiento antidepresivo y sus efectos se mantienen a largo plazo (14-15).

Ante esta situación, se hace necesario conocer la situación concreta de nuestro ámbito de trabajo. Por ello se propone realizar una investigación sobre la demanda y la respuesta asistencial de Salud Mental con la finalidad de obtener información que sirva para la planificación e implementación de programas asistenciales específicos que permitan una atención de calidad a los trastornos más prevalentes sin perjuicio de los más graves. Además, se pretende afianzar la coordinación entre niveles asistenciales con el fin de mejorar el proceso de derivación y una atención ajustada a las necesidades y demandas de los usuarios de Salud Mental.

Tras una revisión bibliográfica se ha detectado que no existen publicaciones científicas sobre análisis de la derivación y demanda en los Servicios de Salud 
Mental en el Principado de Asturias. En este trabajo presentamos los resultados del análisis de la demanda realizada en tres CSM. Tiene como objetivos describir las características de la demanda así como analizar el tipo de respuesta asistencial proporcionada desde nuestros servicios.

\section{Método}

\section{Muestra}

La muestra se toma de aquellos usuarios derivados a tres CSM del Servicio de Salud del Principado de Asturias entre los meses de febrero y mayo de 2012. Se contabilizan un total de 492 derivaciones y se recogen datos de 381 personas, habiendo por tanto 111 sujetos perdidos. Un 95,9\% de los pacientes vienen derivados desde Atención Primaria, un 1,9\% de urgencias, un 1,6\% de otras especialidades médicas (por ejemplo Medicina Interna o Endocrinología) y finalmente un 0,6\% procede de otros dispositivos diferentes a los mencionados.

\section{Variables e instrumentos}

Se elaboró un cuestionario construido ad hoc para la recogida de la información. Algunas de las variables estaban previamente categorizadas y otras se categorizaron a posteriori en función de la información obtenida.

Se recogieron las siguientes variables:

- Variables sociodemográficas.

Se toman de la historia clínica que se abre en el CSM correspondiente y del volante de derivación.

- Variables de la demanda.

La información se recoge del volante de derivación y son las siguientes:

- Origen de la derivación, es decir, quién es el derivante.

- Tipo de demanda. Se tiene en cuenta si es ordinaria, preferente o urgente.

- Tarea delegada en el volante. Se trata de aquella tarea que el derivante solicita a Salud Mental, por ejemplo diagnóstico o valoración.

- Indicación de profesional por parte del derivante. El derivante puede solicitar psicólogo clínico, psiquiatra o que la demanda vaya dirigida a Salud Mental. Se excluyen los retornos ya que generalmente se deriva al profesional que atendió previamente al paciente y esto podría sesgar los resultados.

- Variables de la respuesta asistencial.

Se toman de la historia clínica del CSM.

- Profesional asignado en el CSM. Se trata de aquel profesional que toma a cargo al paciente, al menos inicialmente, ya sea psicólogo clínico o psiquiatra. Se excluyen los retornos por el mismo motivo que en la variable indicación de profesional. 
ORIGINALES Y REVISIONES

- Asistencia a primera consulta. Se valora si el paciente acudió a la primera consulta en el CSM o no.

- Variables clínicas.

Valoradas mediante el análisis de la historia clínica y del volante de derivación.

- Motivo de derivación. La información se tomó del volante de derivación y las categorías de esta variable se llevaron a cabo a posteriori, una vez recogidos todos los datos.

- Antecedentes asistenciales en Salud Mental. Se tuvo en cuenta si el paciente tenía antecedentes o no, y si estos eran en atención pública o privada.

- Diagnóstico según el volante. Se trata de la impresión diagnóstica del derivante. En la mayoría de los casos no coinciden con los sistemas de clasificación DSM y CIE ya que son diagnósticos procedentes del sistema informático de Atención Primaria. Por este motivo pueden aparecer diagnósticos que en Salud Mental no se consideran como tales, como por ejemplo problemas interpersonales. Dada la variedad de diagnósticos que aparecen en los volantes, se organizaron estos en categorías más amplias tales como ansiedad, depresión, trastorno adaptativo, etc.

En una segunda parte del estudio se aplicó a los usuarios un cuestionario de preferencias, expectativas e información recibida por parte del derivante.

\section{Procedimiento}

Se trata de un estudio descriptivo observacional llevado a cabo en tres centros: CSM La Ería (Oviedo), CSM La Calzada (Gijón) y CSM Llano Ponte (Avilés). En cada CSM había un profesional responsable de la recogida de la información.

El estudio cuenta con la aprobación de las tres direcciones de Área de Gestión Clínica y del Comité Ético de Investigación Clínica Regional del Principado de Asturias.

Al llegar una nueva derivación se tomaban los datos del volante y de la historia clínica; en el caso de Gijón y Avilés se trata de historia electrónica y en Oviedo es historia en papel. Posteriormente el responsable decidía si el paciente podía incluirse en la segunda parte del estudio, es decir, en la aplicación del cuestionario de preferencias, expectativas e información recibida por parte del derivante. En caso de que así fuera, trataba de contactar con el usuario vía telefónica.

Una vez recogidos todos los datos, se transcriben y analizan mediante el paquete estadístico SPSS v.17. Se realizan pruebas de estadística descriptiva con el fin de caracterizar la distribución de las diferentes variables, así como pruebas de Chi Cuadrado para comprobar la relación entre variables nominales. 


\section{Resultados}

- Variables sociodemográficas.

En la siguiente tabla se exponen las variables sociodemográficas de la muestra. Dadas las características de los diferentes centros en los que se llevó a cabo la investigación, resultó imposible recoger información del 100\% de los sujetos, por ello se especifica el porcentaje de datos obtenidos.

Tabla1.

Variables sociodemográficas de la muestra.

\begin{tabular}{|c|c|c|}
\hline Variable sociodemográfica & Valor & Datos obtenidos $(\%)$ \\
\hline $\begin{array}{l}\text { Edad } \\
\text { Rango } \\
\text { Media } \\
\text { Desviación típica }\end{array}$ & $\begin{array}{l}15-97 \\
44,07 \\
17,59\end{array}$ & $98,7 \%$ \\
\hline $\begin{array}{l}\text { Sexo } \\
\text { Mujeres } \\
\text { Hombres } \\
\end{array}$ & $\begin{array}{l}58,5 \%(n=220) \\
41,5 \%(n=156)\end{array}$ & $98,7 \%$ \\
\hline $\begin{array}{l}\text { Convivencia } \\
\text { Familia origen } \\
\text { Familia propia } \\
\text { Solo } \\
\text { Con otras personas } \\
\text { Institución o piso protegido }\end{array}$ & $\begin{array}{c}26,3 \%(\mathrm{n}=78) \\
53,9 \%(\mathrm{n}=160) \\
15,8 \%(\mathrm{n}=47) \\
3,4 \%(\mathrm{n}=10) \\
0,7 \%(\mathrm{n}=2) \\
\end{array}$ & $78 \%$ \\
\hline $\begin{array}{l}\text { Estado civil } \\
\text { Casado o en pareja } \\
\text { Soltero } \\
\text { Separado o divorciado } \\
\text { Viudo }\end{array}$ & $\begin{array}{c}53,5 \%(\mathrm{n}=162) \\
28,7 \%(\mathrm{n}=87) \\
11,2 \%(\mathrm{n}=34) \\
6,6 \%(\mathrm{n}=20)\end{array}$ & $79,5 \%$ \\
\hline $\begin{array}{l}\text { Situación laboral } \\
\text { Activo } \\
\text { Parado } \\
\text { Jubilado o pensionista } \\
\text { Estudiante } \\
\text { Labores del hogar } \\
\text { Incapacidad laboral temporal }\end{array}$ & $\begin{array}{c}36,7 \%(\mathrm{n}=99) \\
17,4 \%(\mathrm{n}=47) \\
14,4 \%(\mathrm{n}=39) \\
10,4 \%(\mathrm{n}=28) \\
7 \%(\mathrm{n}=19) \\
14,1 \%(\mathrm{n}=38)\end{array}$ & $70,9 \%$ \\
\hline $\begin{array}{l}\text { Medio } \\
\text { Urbano } \\
\text { Rural }\end{array}$ & $\begin{array}{c}77,6 \%(n=225) \\
22,4 \%(n=65)\end{array}$ & $76,1 \%$ \\
\hline
\end{tabular}

- Variables de la demanda.

- Respecto al origen de la derivación, como ya se mencionó anteriormente, la gran mayoría de las demandas procede de Atención Primaria (95,9\%).

- En cuanto a la tarea delegada en el volante, el 80,4\% $(n=255)$ de los derivantes solicitan diagnóstico y tratamiento (no implica que sea tratamiento farmacológico), el 7,9\% $(n=25)$ valoración, el 2,5\% $(n=8)$ psicoterapia y finalmen- 
te el 4,3\% (n=14) de los derivantes solicitan otras tareas como seguimiento o revisión farmacológica. En un 4,7\% $(n=15)$ de los volantes no consta la tarea delegada en Salud Mental. Esta información corresponde al 83,2\% de los volantes $(\mathrm{n}=317)$.

- Un 45,6\% ( $\mathrm{n}=113)$ de los derivantes solicitan psiquiatra, un 20,2\% ( $\mathrm{n}=50)$ psicólogo clínico y un 32,7\% $(\mathrm{n}=81)$ realizan una demanda genérica a Salud Mental. Un $0,8 \%(\mathrm{n}=2)$ piden ambos profesionales y asimismo en un $0,8 \%$ $(\mathrm{n}=2)$ de las demandas no consta el profesional solicitado. Obtuvimos información de un $86,71 \%$ de los casos ( $\mathrm{n}=248$ ).

- Con respecto al tipo de consulta solicitada, en el 76,9\% $(\mathrm{n}=257)$ de los casos se trata de una demanda ordinaria, el $22,2 \%(n=74)$ es preferente y se realizan un $0,9 \%(\mathrm{n}=3)$ de consultas urgentes. Se recogió información del $87,66 \%$ de las demandas $(\mathrm{n}=334)$.

- Variables de la respuesta asistencial.

- Respecto al profesional asignado a la demanda, un 59,1\% ( $\mathrm{n}=152)$ de las demandas son asignadas a psiquiatra y un $39,7 \%(n=102)$ a psicólogo clínico. En un caso se asigna directamente a ambos profesionales. Son datos del $89,16 \%$ de los casos, excluyendo retornos $(\mathrm{n}=255)$.

- Un 89,9\% $(\mathrm{n}=240)$ de los pacientes acude a la primera consulta, mientras que un $10,1 \%(n=27)$ no lo hacen.

- Variables clínicas.

- En cuanto a antecedentes asistenciales, un $55,9 \%(\mathrm{n}=152)$ no presentaban antecedentes en atención especializada, un 29\% $(n=79)$ habían sido atendidos a nivel ambulatorio en los Servicios de Salud Mental públicos y un 9,6\% $(\mathrm{n}=26)$ recibieron asistencia ambulatoria privada. Un 5,4\% $(n=15)$ tenía otro tipo de antecedentes diferentes a los anteriores, como por ejemplo atención en urgencias psiquiátricas. Esta información es relativa al 71,39\% de los casos $(\mathrm{n}=272)$.

- Respecto al motivo de derivación que aparece en el volante, un 40,06\% $(\mathrm{n}=127)$ de los pacientes son derivados por presentar síntomas o trastorno de Salud Mental en relación a circunstancias psicosociales y/o enfermedad somática (por ejemplo, un duelo o diagnóstico de cáncer) y un 33,9\% $(\mathrm{n}=108)$ por síntomas o un trastorno de Salud Mental (por ejemplo, ansiedad o apatía). Otros motivos de derivación menos frecuentes fueron los siguientes: problemas psicosociales $(3,8 \%)$, problemas de comportamiento $(3,1 \%)$, revisión $(3,1 \%)$, recomendación por parte de otro servicio privado o público de acudir a Salud Mental (2,5\%), solicitud por parte del propio paciente o alguien de su entorno (2,2\%). Un $9,7 \%$ de los pacientes son derivados por otros motivos diferentes a los anteriores. Es importante aclarar que el motivo de derivación que destaca el derivante en el volante de derivación puede no ser el único, es decir, el que enfatice la presencia de conflictos psicosociales no implica que 
no exista sintomatología. Por otra parte, el que haga referencia a síntomas o a un cuadro psiquiátrico no quiere decir que no existan precipitantes en el entorno. Los datos corresponden al $83,2 \%(n=317)$ de los casos y son los sujetos que aportaron el volante de derivación.

- En lo que se refiere al diagnóstico reflejado en el volante de derivación, encontramos un 30\% $(n=95)$ de casos de ansiedad, un 23\% $(n=73)$ de depresión, un $11,4 \%(\mathrm{n}=36)$ de trastorno adaptativo, un $5 \%(\mathrm{n}=16)$ de alteración del comportamiento y un $4,4 \%(n=14)$ de trastorno ansioso-depresivo. Otros diagnósticos menos frecuentes fueron los siguientes: TOC (2,5\%), consumo de drogas $(2,2 \%)$, trastorno psicótico $(1,9 \%)$, alteración de la personalidad $(1,6 \%)$, alteraciones del sueño $(1,6 \%)$, problemas de alimentación $(1,6 \%)$, problemas interpersonales $(1,6 \%)$ y deterioro cognitivo $(0,6 \%)$. En un $6 \%$ de los casos no consta el diagnóstico y en un $6,6 \%$ se trata de otros diagnósticos como disfunciones sexuales o ideación autolítica.

- Pruebas de Chi Cuadrado $\left(\chi^{2}\right)$.

- Existe relación entre el profesional demandado por parte del derivante y el profesional asignado en el CSM $\left(\chi^{2}=69,160, p=0,000\right)$.

Tabla 2 .

Relación entre el profesional demandado y el profesional asignado.

\begin{tabular}{|l|l|c|c|c|c|}
\hline Profesional demandado & & $\begin{array}{c}\text { Asignación Psicólogo } \\
\text { Clínico }\end{array}$ & $\begin{array}{c}\text { Asignación } \\
\text { Psiquiatra }\end{array}$ & $\begin{array}{c}\text { No consta } \\
\text { asignación }\end{array}$ & Total \\
\hline Psicólogo Clínico & $\mathrm{n}$ & 42 & 8 & 0 & - \\
Psiquiatra & $\%$ & $84 \%$ & $16 \%$ & 0 & 113 \\
& $\mathrm{n}$ & 22 & 91 & - & 81 \\
Salud Mental & $\%$ & $19,46 \%$ & $80,53 \%$ & 2 & 2 \\
No consta & $\mathrm{n}$ & 33 & 46 & $2,47 \%$ & 0 \\
Ambos & $\%$ & $40,74 \%$ & $26,79 \%$ & - & 2 \\
\hline Total & $\mathrm{n}$ & 0 & $100 \%$ & - & 248 \\
\hline
\end{tabular}

$\chi^{2}=69,160 ; p=0,000$.

- Se constatan diferencias estadísticamente significativas en la asignación del profesional en función del diagnóstico $\left(\chi^{2}=46,995, \mathrm{p}=0,014\right)$, significación influida en gran parte por una mayor asignación de casos de depresión a psiquiatra. También los casos de trastorno ansioso-depresivo, trastorno adaptativo y TOC son atendidos en primera consulta en mayor medida por psiquiatras. Casos catalogados como alteración del comportamiento se asignan en mayor porcentaje a psicólogos clínicos. 
Tabla 3.

Asignación de profesional en función del diagnóstico.

\begin{tabular}{|c|c|c|c|c|c|c|}
\hline Diagnóstico & & $\begin{array}{c}\text { Asignación Ps. } \\
\text { Clínico }\end{array}$ & $\begin{array}{c}\text { Asignación } \\
\text { Psiquiatra }\end{array}$ & No Consta & Ambos & Total \\
\hline Ansiedad & $\begin{array}{l}\mathrm{n} \\
\%\end{array}$ & $\begin{array}{c}36 \\
50 \%\end{array}$ & $\begin{array}{c}35 \\
48,61 \%\end{array}$ & $\begin{array}{c}1 \\
1,38 \%\end{array}$ & $\begin{array}{l}0 \\
-\end{array}$ & 72 \\
\hline Depresión & $\begin{array}{l}\mathrm{n} \\
\%\end{array}$ & $\begin{array}{c}9 \\
15,51 \%\end{array}$ & $\begin{array}{c}49 \\
84,48 \%\end{array}$ & 0 & $\begin{array}{l}0 \\
-\end{array}$ & 58 \\
\hline T. ansioso-depresivo & $\begin{array}{l}\mathrm{n} \\
\%\end{array}$ & $\begin{array}{c}5 \\
38,36 \%\end{array}$ & $\begin{aligned} 8 \\
61,53 \%\end{aligned}$ & $\begin{array}{l}0 \\
-\end{array}$ & $\begin{array}{l}0 \\
-\end{array}$ & 13 \\
\hline T. Adaptativo & $\begin{array}{l}\mathrm{n} \\
\%\end{array}$ & $\begin{array}{c}12 \\
41,37 \%\end{array}$ & $\begin{array}{c}17 \\
58,62 \%\end{array}$ & $\begin{array}{l}0 \\
-\end{array}$ & $\begin{array}{l}0 \\
-\end{array}$ & 29 \\
\hline Alteración comportamiento & $\begin{array}{l}\mathrm{n} \\
\%\end{array}$ & $\begin{array}{c}8 \\
57,14 \%\end{array}$ & $\begin{array}{c}5 \\
35,71 \%\end{array}$ & $\begin{array}{c}1 \\
7,14 \%\end{array}$ & $\begin{array}{l}0 \\
-\end{array}$ & 14 \\
\hline Consumo de sustancias & $\begin{array}{l}\mathrm{n} \\
\%\end{array}$ & $\begin{array}{c}1 \\
33,33 \%\end{array}$ & $\begin{array}{c}2 \\
66,66 \%\end{array}$ & $\begin{array}{l}0 \\
-\end{array}$ & $\begin{array}{l}0 \\
-\end{array}$ & 3 \\
\hline Alteración personalidad & $\begin{array}{l}\mathrm{n} \\
\%\end{array}$ & $\begin{array}{c}3 \\
75 \%\end{array}$ & $\begin{array}{c}1 \\
25 \%\end{array}$ & $\begin{array}{l}0 \\
-\end{array}$ & $\begin{array}{l}0 \\
-\end{array}$ & 4 \\
\hline Alteración del sueño & $\begin{array}{l}\mathrm{n} \\
\%\end{array}$ & $\begin{array}{c}2 \\
50 \%\end{array}$ & $\begin{array}{c}2 \\
50 \%\end{array}$ & $\begin{array}{l}0 \\
-\end{array}$ & $\begin{array}{l}0 \\
-\end{array}$ & 4 \\
\hline Problema de alimentación & $\begin{array}{l}\mathrm{n} \\
\%\end{array}$ & $\begin{array}{c}2 \\
50 \%\end{array}$ & $\begin{array}{c}2 \\
50 \%\end{array}$ & $\begin{array}{l}0 \\
-\end{array}$ & $\begin{array}{l}0 \\
-\end{array}$ & 4 \\
\hline TOC & $\begin{array}{l}\mathrm{n} \\
\%\end{array}$ & $\begin{array}{l}0 \\
-\end{array}$ & $\begin{array}{c}6 \\
100 \%\end{array}$ & $\begin{array}{l}0 \\
-\end{array}$ & $\begin{array}{l}0 \\
-\end{array}$ & 6 \\
\hline Problema interpersonal & $\begin{array}{l}\mathrm{n} \\
\%\end{array}$ & $\begin{array}{c}3 \\
100 \%\end{array}$ & $\begin{array}{l}0 \\
-\end{array}$ & $\begin{array}{l}0 \\
-\end{array}$ & $\begin{array}{l}0 \\
-\end{array}$ & 3 \\
\hline Trastorno psicótico & $\begin{array}{l}\mathrm{n} \\
\%\end{array}$ & $\begin{array}{l}0 \\
-\end{array}$ & $\begin{array}{c}2 \\
66,66 \%\end{array}$ & $\begin{array}{l}0 \\
-\end{array}$ & $\begin{array}{c}1 \\
33,33 \%\end{array}$ & 3 \\
\hline No Consta & $\begin{array}{l}\mathrm{n} \\
\%\end{array}$ & $\begin{array}{c}7 \\
43,75 \%\end{array}$ & $\begin{array}{c}9 \\
56,25 \%\end{array}$ & $\begin{array}{l}0 \\
-\end{array}$ & $\begin{array}{l}0 \\
-\end{array}$ & 16 \\
\hline Otros & $\begin{array}{l}\mathrm{n} \\
\% \\
\end{array}$ & $\begin{array}{c}11 \\
64,7 \% \\
\end{array}$ & $\begin{array}{c}6 \\
35,29 \% \\
\end{array}$ & $\begin{array}{l}0 \\
-\end{array}$ & $\begin{array}{l}0 \\
-\end{array}$ & 17 \\
\hline Total & $\mathrm{N}$ & 99 & 144 & 2 & 1 & 246 \\
\hline
\end{tabular}

$\chi^{2}=46,995 ; \mathrm{p}=0,014$.

\section{Discusión}

A la luz de estos resultados cabe destacar varias implicaciones del estudio. En primer lugar se constata la elevada frecuencia de trastornos mentales comunes, aproximadamente del $68,8 \%$. Además, podemos observar que no se cumplen las recomendaciones de guías clínicas dado que se asignan a psiquiatra mayoritariamente casos de depresión, y también existe una mayor asignación de trastornos ansiosodepresivos y trastornos adaptativos. No parecen existir diferencias en cuanto a trastornos de ansiedad (recordemos que el tratamiento de elección es psicológico).

Sin embargo, esto no implica que los psicólogos clínicos reciban un porcentaje significativamente menor de derivaciones. Los CSM asignan a consulta de psicología clínica un porcentaje del 39,7\% de las primeras demandas, lo que significa que la atención psicológica se considera necesaria para las demandas de 
muchos usuarios que presentan problemas de salud mental. Este hecho contrasta, sin embargo, con la distribución de los equipos, dado que existe un número considerablemente menor de psicólogos clínicos en los mismos.

En términos generales se puede decir que la respuesta asistencial del CSM coincide con la petición del derivante. Este dato en principio parece algo positivo, pero habría que valorar qué pautas han utilizado los derivantes para realizar dicha recomendación.

A partir de los datos resultaría fundamental definir unos criterios de derivación a Salud Mental así como respecto a la asignación a diferentes profesionales, dado que las prestaciones asistenciales no son las mismas. Estos criterios deberían tener en cuenta las recomendaciones de guías clínicas y de la Estrategia en Salud Mental del Sistema Nacional de Salud 2009-2013 (1), dado que uno de sus objetivos es facilitar el acceso de la población a intervenciones psicoterapéuticas.

Otra cuestión a considerar es que que muchos de los casos que se derivan como trastornos mentales comunes pueden corresponder en realidad con códigos $Z$, sobre todo si tenemos en cuenta que en un $40,06 \%$ de las derivaciones la sintomatología está asociada a circunstancias vitales estresantes. Este resultado pone de manifiesto la dificultad que supone para Atención Primaria dar una respuesta adecuada a este tipo de demandas sólo desde el enfoque farmacológico, dado que son derivados a la atención especializada. En estos casos la intervención debería ser fundamentalmente preventiva, con el objetivo de evitar que determinadas situaciones psicosociales den lugar a dinámicas disfuncionales. Para estas demandas y también para casos leves de trastornos mentales comunes sería fundamental introducir en Atención Primaria la figura del psicólogo clínico y la atención psicoterapéutica. Esto permitiría evitar la sobrecarga de la atención especializada, prevenir la aparición de trastornos de entidad clínica y proporcionar a los usuarios la asistencia que demandan, estando caracterizada esta por su brevedad y eficiencia.

En definitiva, ante estos resultados planteamos la importancia del Psicólogo Clínico y de la psicoterapia tanto en Salud Mental como en Atención Primaria, con los objetivos de reducir la carga asistencial y la cronificación asociada al tratamiento de trastornos mentales comunes y códigos Z. Sin embargo, para que las recomendaciones puedan cumplirse sería necesario un mayor desarrollo de la atención psicológica en nuestro país, y especialmente en nuestra Comunidad Autónoma (16).

Por otra parte es necesario tener en cuenta las múltiples limitaciones que presenta el estudio y que hacen preciso tomar con cautela los datos. La investigación se realizó en tres áreas sanitarias, entre las cuales pueden existir diferencias a pesar de que la estructura de los centros y el sistema de derivación es similar. Dado que no disponíamos de información suficiente para analizar los datos de forma independiente, es posible que el tomarlos en conjunto haya supuesto una fuente de sesgo. Hubiese sido fundamental comprobar que no existían diferencias entre las 
ORIGINALES Y REVISIONES

áreas pero, como ya se mencionó, el limitado número de sujetos por zona impedía realizar esta comparación de forma fiable.

Otra cuestión es la pérdida de información en el proceso de recogida de la misma. Dados los diferentes sistemas de codificación y el hecho de recoger en muchas ocasiones datos tomados por otros profesionales ajenos al estudio, en un amplio porcentaje de los casos no se pudo obtener información de determinadas variables. También dependíamos de que el paciente trajese el volante de derivación en las primeras consultas, lo cual en muchos casos no era así.

El método de selección de la muestra también es un aspecto a mejorar. Los pacientes se iban incluyendo en el estudio según llegaban las derivaciones al CSM en un determinado periodo de tiempo. Por tanto la selección de la muestra no se hizo de forma aleatorizada y esto supone un sesgo para la representatividad de la misma. Además pueden existir efectos de estacionalidad de la demanda.

Por último, los diagnósticos que se utilizaron fueron aquellos valorados por el MAP. Dado que en Atención Primaria no se lleva a cabo una evaluación tan exhaustiva como en Salud Mental, es posible que estos diagnósticos sean imprecisos. Sin embargo, la asignación a cada profesional se realiza en base a la información proporcionada por el MAP, por lo que se consideró relevante tenerlos en cuenta.

Para concluir consideramos que este estudio puede ser un primer paso para empezar a considerar la calidad de la asistencia especializada en Salud Mental en nuestros servicios. Dado el momento histórico de crisis económica y social que vivimos, cobra aún más importancia el buscar soluciones que mejoren la eficiencia y la satisfacción de los usuarios. Las intervenciones psicológicas especializadas cumplen con ambos requisitos $(7,13)$, pero únicamente si los profesionales están en disposición de ofrecer una atención de calidad que responda a las necesidades de los usuarios y los derivantes.

\section{BIBLIOGRAFÍA}

(1) Estrategia en Salud Mental Sistema Nacional de Salud 2009-2013. Ministerio de Sanidad, Política Social e Igualdad, 2011. Disponible en http://www.msps.es/organizacion/sns/planCalidadSNS/docs/saludmental/SaludMental2009-2013.pdf

(2) García J. Las psicoterapias en los servicios públicos de Salud Mental. ABACO. 1990, 8: 56-60.

(3) Servicio de Salud del Principado de Asturias, SESPA. Plan Estratégico para el Desarrollo de los Servicios de Salud Mental, 2001-2003.

(4) Servicio de Salud del Principado de Asturias, SESPA. Revisión y Actualización del Plan Estratégico para el Desarrollo de los Servicios de Salud Mental, 2005-2007.

(5) Ortiz A, González R, y Rodríguez F. La derivación a Salud Mental de pacientes sin trastorno psíquico diagnosticable. Atención Primaria. 2006, 38 (10): 563-569. 
(6) Estrategia en Salud Mental del Sistema Nacional de Salud. Ministerio de Sanidad y Consumo, 2007.

(7) Pérez Álvarez M, Fernández Hermida JR, Fernández Rodríguez C y Amigo Vázquez I. Guía de tratamientos psicológicos eficaces I. Adultos. Madrid, Pirámide 2003.

(8) Pérez Álvarez M, Fernández Hermida JR, Fernández Rodríguez C y Amigo Vázquez I. Guía de tratamientos psicológicos eficaces II. Psicología de la Salud. Madrid, Pirámide 2003.

(9) Pérez Álvarez M, Fernández Hermida JR, Fernández Rodríguez C y Amigo Vázquez I. Guía de tratamientos psicológicos eficaces III. Infancia y Adolescencia. Madrid, Pirámide 2003.

(10) Dimidjian S, Hollon SD, Dobson KS, Schmaling KB, Kohlenberg RJ, Addis ME, Gallop R, McGlinchey JB, Markley DK, Gollan JK, Atkins DC, Dunner DL \& Jacobson NS. Randomized Trial of Behaviorial Activation, Cognitive Therapy, and Antidepressant Medication in the Acute Treatment of Adult. J Consult Clin Psych. 2006, 74 (4), 658-670.

(11) National Institute for Health and Clinical Excellence (NICE). Clinical Guideline 22. Anxiety: management of anxiety (panic disorder, with or without agoraphobia, and generalised anxiety disorder) in adults in primary, secondary and community care. London, National Institute for Health and Clinical Excellence 2004.

(12) Consejería de Salud y Servicios Sanitarios. Programas Clave de Atención Multidisciplinar. Guía de Recomendaciones Clínicas. Ansiedad. Oviedo, Gobierno del Principado de Asturias, 2006.

(13) Unidad de Evaluación de Tecnologías Sanitarias. Agencia Laín Entralgo. Guías de Práctica Clínica en el SNS. Guía de Práctica Clínica para el Manejo de Pacientes con Trastornos de Ansiedad en Atención Primaria. Madrid, MSC 2008.

(14) Consejería de Salud y Servicios Sanitarios. Programas Clave de Atención Multidisciplinar. Guía de Recomendaciones Clínicas. Depresión. Oviedo, Gobierno del Principado de Asturias 2008.

(15) Nacional Institute for Health and Clinical Excellence (NICE). Clinical Guideline 90. The treatment and management of depression in adults. London, National Institute for Health and Clinical Excellence 2009.

(16) Jiménez Estévez JF. El papel del Observatorio de Salud Mental y los criterios de calidad asistencial de la AEN en la evaluación y planificación asistencial. Recursos de profesionales en CSM y de dispositivos de atención a pacientes con TMG en España. Rev. Asoc. Esp. Neuropsiq. 2011, vol. 31 no. 4 Madrid oct.-dic. 